\title{
Impact of diabetes on hospital admission and length of stay among a general population aged 45 year or more: a record linkage study
}

Elizabeth Jean Comino ${ }^{1 *}$, Mark Fort Harris ${ }^{1}$, MD Fakhrul Islam', Duong Thuy Tran², Bin Jalaludin 3,4, Louisa Jorm ${ }^{2,5}$, Jeff Flack ${ }^{6}$ and Marion Haas ${ }^{7}$

\begin{abstract}
Background: The increased prevalence of diabetes and its significant impact on use of health care services, particularly hospitals, is a concern for health planners. This paper explores the risk factors for all-cause hospitalisation and the excess risk due to diabetes in a large sample of older Australians.

Methods: The study population was 263,482 participants in the 45 and Up Study. The data assessed were linked records of hospital admissions in the 12 months following completion of a baseline questionnaire. All cause and ambulatory care sensitive admission rates and length of stay were examined. The associations between demographic characteristics, socioeconomic status, lifestyle factors, and health and wellbeing and risk of hospitalisation were explored using zero inflated Poisson (ZIP) regression models adjusting for age and gender. The ratios of adjusted relative rates and $95 \%$ confidence intervals were calculated to determine the excess risk due to diabetes.

Results: Prevalence of diabetes was $9.0 \%(n=23,779)$. Age adjusted admission rates for all-cause hospitalisation were 631.3 and 454.8 per 1,000 participant years and the mean length of stay was 8.2 and 7.1 days respectively for participants with and without diabetes. In people with and without diabetes, the risk of hospitalisation was associated with age, gender, household income, smoking, BMl, physical activity, and health and wellbeing. However, the increased risk of hospitalisation was attenuated for participants with diabetes who were older, obese, or had hypertension or hyperlipidaemia and enhanced for those participants with diabetes who were male, on low income, current smokers or who had anxiety or depression.

Conclusions: This study is one of the few studies published to explore the impact of diabetes on hospitalisation in a large non-clinical population, the 45 and Up Study. The attenuation of risk associated with some factors is likely to be due to correlation between diabetes and factors such as age and obesity. The increased risk in association with other factors such as gender and low income in participants with diabetes is likely to be due to their synergistic influence on health status and the way services are accessed.
\end{abstract}

Keywords: Healthy aging, Diabetes, Hospital admission, Record linkage, Cohort study, Socioeconomic status, Health and wellbeing

\footnotetext{
* Correspondence: E.Comino@unsw.edu.au

${ }^{1}$ Centre for Primary Health Care and Equity, University of New South Wales,

Sydney, NSW 2052, Australia

Full list of author information is available at the end of the article
} 


\section{Background}

Diabetes mellitus is a serious public health problem that has implications for individuals, communities, and health and human services [1]. The rapid increase in the prevalence of diabetes, driven by the increased prevalence of obesity and an aging population, has led to it being described as an epidemic. Diabetes comprises a complex of metabolic disorders associated with impaired insulin secretion and glucose metabolism [2,3]. Among older people, diabetes is associated with poor health outcomes including hyperglycaemia, increased cardiovascular risk, and peripheral vascular problems, and is associated with increased use of health services including unplanned hospitalisation and premature mortality $[3,4]$. The importance of early detection and management of diabetes to prevent disease progression, poor health outcomes including early onset of complications, and increased use of health services is recognised and supported by policy and practice interventions to improve diabetes care. Yet diabetes remains a significant reason for preventable contact with the health system $[5,6]$.

A number of studies have demonstrated that people with diabetes [5,7-10] have hospital admission rates between 2 and 6 times higher than people without diabetes [5,9-11]. People with diabetes also have excessive lengths of hospital stay compared to people without diabetes $[5,9,11]$. These previous studies used hospital or practice-based populations $[5,9,11]$. Study of hospital-based populations may represent people with severe diabetes including complications of diabetes and its associated morbidity. As results, the associated risks as well as hospitalisation rates could be over-estimated. There is a need to determine the risk of hospitalisation and impact of diabetes among a general community population.

It is generally accepted that risk factors such as age, gender, education, socioeconomic status, lifestyle risk factors, and health status are associated with poorer health outcomes including hospitalisation among the general population. This is no less so for people with diabetes where lower socioeconomic status, older age, obesity, tobacco smoking, physical inactivity, poor glycaemic control and clinical indicators including glycosylated haemoglobin (HbA1c), insulin use, longer duration of diabetes, and presence of complications have been associated with increased rates of hospitalisation and longer length of stay $[7,12,13]$. Few studies have examined these associations for a community dwelling population of people with and without diabetes.

The establishment of a large population based cohort of New South Wales, Australia residents aged 45 year and older at recruitment and enhanced linkage facilities enabled us to create a community dwelling population study of diabetes. In this paper we describe the association between hospital admission and wide range of demographic characteristics, socioeconomic status, lifestyle, and health and wellbeing among study participants with and without diabetes. We also explore the excess risk of hospitalisation that may be attributed to diabetes.

\section{Methods}

Data were obtained from the following sources:

\section{The Sax institute: $\mathbf{4 5}$ and Up study}

The Sax Institute's 45 and Up Study is a cohort study of more than 250,000 residents of NSW, Australia. Recruitment of this cohort has been described elsewhere [14]. Briefly, potential study participants comprised progressive random samples of adults aged 45 years or older registered on the Medicare Australia (a universal health insurance scheme) database held by the Department of Human Services. Participants joined the Study by completing a baseline questionnaire and providing consent for long-term follow up including linkage of their baseline data to their health records. Recruitment was undertaken between February 2006 and April 2009.

The baseline questionnaire collected information on a range of participant characteristics (available at www.45andup.org.au). Our method of identifying participants with diabetes at baseline is described elsewhere [15]. The majority of participants was defined as having diabetes based on their response to Question 24: 'Has a doctor EVER told you that you have diabetes?'. Other diabetic participants were identified on the basis of free-text responses to a question eliciting current important illnesses and self-reported use of diabetes medications. The baseline survey did not differentiate type of diabetes. We included all participants identified as having diabetes.

Demographic variables (age, date of recruitment, gender, country of birth), socioeconomic status (highest education level, household income), and lifestyle (smoking status, alcohol intake, body mass index (BMI), and physical activity) were obtained from the baseline questionnaire. BMI $\left(\mathrm{Kg} / \mathrm{M}^{2}\right)$ was classified as normal weight $(<25)$, overweight (25-29), and obese ( $>30)$. Physical activity was classified as sedentary if no physical activity was reported and sufficient if physical activity comprised at least 150 minutes of walking during at least 5 sessions per week; all other categories were classified as insufficient [16].

Socioeconomic measures of residential areas included the Australian Bureau of Statistics Index of Relative Socioeconomic Disadvantage (SEIFA IRSD) [17] and remoteness according to the mean Accessibility Remoteness Index of Australia Plus (ARIA+) score for the Statistical Local Area of residence [18]. SEIFA IRSD was classified as quintile where 1 was the least and 5 the most disadvantaged. ARIA+ was classified as major city, inner regional, and outer regional and remote (grouped). Health status was measured in a number 
of ways. Self-rated general health and quality of life were self-rated as excellent or very good, good, and fair or poor. Psychological distress based on the Kessler-10 score [19] was categorised as low (score of 10-15), moderate (16-21), high (22-29) and very high (30-50). Functional capacity was measured using the Medical Outcomes Study, Short Form 36 Physical Functioning Scale (SF36-PF) [20] and classified as no limitation (score of 100), minor (90-99), moderate (60-89) and severe (0-59). The choice of cut-off for these scores was based on previous research [21]. The number of chronic conditions was identified from participants' responses to the questions "Has a doctor ever told you that you have...." or 'In the last month have you been treated for -?' and listed a number of chronic health conditions including cancer, heart disease, high blood pressure, stroke, anxiety, and depression. Participant responses were summed and classified as none, 1, 2, and 3 or more. Among participants with diabetes, duration of diabetes was calculated from age at diagnosis and age at questionnaire completion.

\section{NSW ministry of health: admitted patient data collection}

Under the Australian health care system, hospital services in New South Wales are provided through a mix of publicly and privately funded health services. The NSW Ministry of Health has responsibility for all inpatient services and collates data on admissions into the NSW Admitted Patient Data Collection (APDC). Data were available for 2000-2009. The APDC collates inpatient admissions (discharges, transfers and deaths) from all public, private, and repatriation hospitals, private day procedure centres and public nursing homes in NSW. These data include demographic characteristics, diagnoses, and length of stay for individual episodes of hospitalisation. The diagnoses were coded using International Classification of Disease 10th revisionAustralian Modification (ICD-10-AM) codes. APDC data were available for this study for 2000-2009. For these analyses, APDC records were extracted for the 12 months following recruitment for each participant.

\section{NSW registry of births, death and marriages: death registrations}

All deaths in NSW are certified as to cause and date by a registered medical practitioner and the certificate registered by the NSW Registry of Births, Death and Marriages (RBDM). These linked data were used to identify participants who died within 12 months of recruitment to the 45 and Up Study.

\section{Record linkage}

Baseline questionnaire data from the 45 and Up study were linked to APDC data for the 12 month period following recruitment to the study and to RBDM to exclude participants who dies during this 12 month period. Record linkage was undertaken by the Centre for Health Record Linkage (CHeReL) according to privacy-preserving protocols and probabilistic methods $[22,23]$. Linked records were assigned a unique 'project person number' which was then returned to the data custodians who released de-identified clinical data to the research team.

Of the 266,771 participants in the 45 and Up Study with baseline information, we excluded 3,289 participants due to linkage errors $(n=24)$, death within 12 months of recruitment $(n=1,632)$, admission for dialysis $(\mathrm{n}=251)$, and uncertain diabetes status $(\mathrm{n}=1,382)$. The final dataset for analysis contained baseline 45 and Up Study data and hospital admission information for 263,482 participants, of whom 23,779 (9.0\%) had diabetes and included 124,035 hospitalisation episodes within 12 months of recruitment, involving 65,777 participants.

\section{Outcome measures}

The primary outcome for this study was any hospitalisation within 12 months following recruitment to the 45 and Up Study. Firstly, participants were categorised as having at least one hospital admission or no hospital admission, and secondly, the aged standardised hospital admission rates (rate of admission per 1,000 participants) were calculated. In addition we estimated for the 12 month period the mean number of hospital admissions, total number of days, and length of stay among participants with and without diabetes.

Using the ICD-10-AM codes, the diagnostic reasons for hospitalisation were categorised as all cause, diabetes where diabetes (E10-E14.9) was the principal reason for admission, metabolic or cardiovascular disease (MCVD), and diabetes related ambulatory care sensitive conditions (ACSCs) [24]. MCVD diagnosis as the principal reason for admission included conditions that were considered to be complications of diabetes (hypersmolarity (E87.0) acidosis (E87.2), transient ischaemic attack (G45), nerve disorders and neuropathies (G50-G64), cataracts and lens disorders $(\mathrm{H} 25-\mathrm{H} 28)$, retinal disorders $(\mathrm{H} 30-\mathrm{H} 36)$, glaucoma (H40-H42), myocardial infarction (I21-I22), other coronary heart diseases (I20, I23-I25), heart failure (I50), stroke and sequelae (I60-I64, I69.0-I69.4), peripheral vascular disease (I70-I74), gingivitis and periodontal disease (K05), kidney diseases (N00-N29) including endstage renal disease (N17-N19), and renal dialysis (Z49)). Diabetes related ACSCs were those admissions where principal diagnosis was diabetes or the principal diagnosis was an MCVD code (listed above) and diabetes was given as an additional diagnosis [24]. Length of stay (days) for each admission was calculated from the dates of admission and discharge, taking into account transfers to other 
facilities during the hospital stay. We calculated the total number of days of hospitalisation during the 12 months and the mean length of stay for each admission.

\section{Statistical analysis}

Descriptive univariate and multivariate methods were used in these analyses. Measures of hospitalisation took into account transfers and same day readmissions. In order to handle the skewed data, we built zero inflated Poisson (ZIP) regression models with a log link function to account for a high proportion of participants who were not admitted during the study period [25]. Adjusted relative rates (aRRs) of hospitalisation (rate per participant year of follow up) with $95 \%$ confidence intervals (95\% CI) were estimated from univariate and multivariate analyses respectively. All multivariate models were adjusted for age and gender. Missing data for each study factor were included in the multivariate model using 'missing values' categories; no variables were excluded due to missing values. We explored the changes in observed associations for hospital admission for all cause, ACSC, and nonelective admissions. It was resolved to use all cause admissions for this study.

Significant interactions were observed between diabetes status and hospitalisation due to the presence of many of the participant characteristics. Consequently, separate models for participants with and without diabetes were built. In order to compare the effect size of the observed associations between participants with and without diabetes, the ratios of adjusted relative rates (Ratio of RR) and their associated 95\% confidence intervals were calculated [26]. A ratio of RR of 1.0 indicates that there is no difference between people with or without diabetes in the strength of the association between the participant characteristic and hospitalisation whereas a value greater than or less than 1.0 indicates enhancement or attenuation of the association between the participant characteristic and hospitalisation. All analyses were carried out in SAS version 9.3 (SAS Institute Inc., Cary, NC, USA). All the tests were two-sided and a p-value of less than or equal to 0.05 was considered statistically significant.

\section{Ethics}

The 45 and Up Study was approved by the University of New South Wales Human Research Ethics Committee. The study was approved by the New South Wales Population and Health Services Research Ethics Committee (HREC/09/ CIPHS/2, 12 May 2009).

\section{Results}

\section{Study sample}

The participant characteristics $(n=263,482)$ by diabetes status are summarised in Table 1. Participants with diabetes were more likely to be male, aged $65-74$ years, born overseas, have not completed year 10 of school, live in a disadvantaged suburb, and to report a household income of less than $\$ 20,000$ compared to participants without diabetes. Participants with diabetes were less likely to drink alcohol but more likely to be ex-smokers, obese, physically inactive, and have heart disease, high blood pressure, high blood cholesterol, depression and anxiety compared to those without diabetes.

\section{Impact of diabetes on hospital admission}

Table 2 summarises the hospital admission data. There were 124,035 admissions among 65,777 participants (25.0\%) who had at least one hospital admission recorded in the 12 months following their recruitment; participants with diabetes were more likely to have at least one admission (32.8\%) than participants without diabetes (24.2\%).

Participants with diabetes $(32.8 \%)$ were more likely to have a hospitalisation than participants without diabetes (24.2\%; aRR: 1.24, 95\% CI: 1.21, 1.26). The age adjusted admission rates for all-cause hospitalisation for participants with and without diabetes were 631.3 and 454.8 per 1,000 participant year respectively (Table 2). The number of hospital days among participants with diabetes (mean (SD): 8.3 (18.6) days, median (min-max): $2(1-472)$ days) were more than those among participants without diabetes (mean (SD): 5.5 (12.4) days, median (min-max): 1 (1 - 476) day) (Table 2). The majority of admissions among those with and without diabetes (61.3\% and $70.1 \%$ respectively) were day only events. Of participants with an admission, $72 \%$ had only one admission. The mean length of stay for admissions of more than one day was 8.2 days (median: 4.8 days) for participants with diabetes and 7.1 days (median: 4 days) for those without diabetes.

The results of regression analyses are presented by diabetes status (Table 3). For participants with and without diabetes, the RR of hospitalisation was higher for male, older age group, Australian-born, lower income, and urban-dwelling participants. Participants living in disadvantaged regions were less likely to have a hospital admission record. Of the lifestyle factors, smoking and sedentary lifestyle were associated with a higher risk of hospitalisation while self-report of higher alcohol consumption was associated with a lower risk. Obesity was associated with hospitalisation for participants with and without diabetes. Health status using both individual and composite measures was also significantly associated with hospitalization for participants with and without diabetes (Table 3).

The adjusted rate ratios for participants with and without diabetes differed significantly for a number of study factors (Table 3). The associations between age, obesity, high blood pressure and high blood cholesterol and hospitalisation were attenuated in people with diabetes compared to those without diabetes. For example, 
Table 1 Demographic, socioeconomic, lifestyle, and health and wellbeing characteristics of participants stratified by diabetes status

\begin{tabular}{|c|c|c|c|}
\hline \multirow{2}{*}{$\begin{array}{l}\text { Demographic } \\
\text { characteristics }\end{array}$} & \multicolumn{2}{|l|}{ Diabetes } & \multirow{2}{*}{$\begin{array}{l}\text { No } \\
\mathrm{n}=2\end{array}$} \\
\hline & $n=23,779$ & $\%$ & \\
\hline \multicolumn{4}{|l|}{ Gender } \\
\hline Male & 13,393 & 56.3 & 108 \\
\hline Female & 10,386 & 43.7 & 131,1 \\
\hline \multicolumn{4}{|c|}{ Age group (years) } \\
\hline $45-59$ & 6,698 & 28.2 & 115,7 \\
\hline 60-74 & 11,143 & 46.9 & 86,1 \\
\hline$\geq 75$ & 5,935 & 25.0 & 37,8 \\
\hline \multicolumn{4}{|c|}{ Duration of diabetes (years) } \\
\hline$<5$ & 7,205 & 30.3 & N/A \\
\hline $5-9$ & 5,670 & 23.8 & N/A \\
\hline $10-14$ & 3,933 & 16.5 & N/A \\
\hline$\geq 15$ & 4,914 & 20.7 & N/A \\
\hline
\end{tabular}

Country of birth

Australia

17,020

Overseas

6,759

$71.6 \quad 180,474$

$28.4 \quad 59,229$

Socioeconomic status

Education

University

Trade/Certificate/Diploma

At least year 10

3,688

$15.5 \quad 57,312$

$30.276,648$

$33.575,707$

Less than Year 10

7,964

$18.426,302$

Household income (\$AUD)

$\geq \$ 70,000$

$\$ 40,000-\$ 69,999$

2,965

3,191

$12.5 \quad 59,4166$

$13.4 \quad 43,588$

$\$ 20,000-\$ 39,999$

4,536

$19.141,579$

$<\$ 20,000$

7,585

$31.9 \quad 43,937$

\section{SEIFA IRSD (quintiles)}

(least disadvantaged) $1^{\text {st }}$

$2^{\text {nd }}$

3,597

$15.1 \quad 51,117$

$17.4 \quad 44,285$

$27.9 \quad 63,680$

$3^{\text {rd }}$

$4^{\text {th }}$

4,148

6,633

5,778

$24.354,034$

$15.2 \quad 26,383$

ARIA+

Major City

Inner Regional

3,611

$15.2 \quad 26,383$

Outer regional/Remote

10,769

$45.3 \quad 107,637$

8,335

$35.1 \quad 84,351$

$19.647,524$

Lifestyle risk factors

Tobacco smoking

status

Never smoked

11,833

10,219

p-value

$\%$

$\begin{array}{ll} & <0.001 \\ 45.3 & \\ 54.7 & \end{array}$

$<0.001$

48.3

35.9

15.8

$<0.001$

$\begin{array}{ll} & \\ & \\ & <0.001 \\ 24.3 & \\ & \\ & \end{array}$

$24.7-2.000$

$<0.001$

23.9

32.0

31.6

11.0

$<0.001$

24.8

18.2

17.4

18.3

$<0.001$

21.3

18.5

26.6

22.5

11.0

0.2

44.9

35.2

19.8

Ex-smoker
$49.8 \quad 136,842$

57.1

35.4
Table 1 Demographic, socioeconomic, lifestyle, and health and wellbeing characteristics of participants stratified by diabetes status (Continued)

\begin{tabular}{lllll}
\hline Current & 1,726 & 7.3 & 17,987 & 7.5
\end{tabular}

Alcohol consumption (standard drinks/week)

$\begin{array}{lllll}0 & 11,073 & 46.6 & 74,732 & 31.2\end{array}$

$\begin{array}{lllll}1-6 & 5,783 & 24.3 & 69,818 & 29.1\end{array}$

$\begin{array}{lllll}7-13 & 2,947 & 12.4 & 45,951 & 19.2\end{array}$

$\begin{array}{lllll}14-20 & 1,846 & 7.8 & 26,881 & 11.2\end{array}$

$\begin{array}{lllll}\geq 20 & 1,445 & 6.1 & 18,308 & 7.6\end{array}$

BMI $\left(\mathrm{kg} / \mathrm{m}^{2}\right)$

$<0.001$

Normal weight $(<25) \quad 4,546 \quad 19.1 \quad 88,806 \quad 37.1$

$\begin{array}{lllll}\text { Overweight (25-29) } \quad 8,083 & 34.0 & 88,255 & 36.8\end{array}$

$\begin{array}{lllll}\text { Obese }(\geq 30) & 9,406 & 39.6 & 47,087 & 19.6\end{array}$

Physical activity**

$\begin{array}{lllll}\text { Sedentary } & 3,084 & 13.0 & 18,191 & 7.6\end{array}$

$\begin{array}{lllll}\text { Insufficient } \quad 5,043 & 21.2 & 41,679 & 17.4\end{array}$

$\begin{array}{lllll}\text { Sufficient } \quad 13,459 & 56.6 & 165,857 & 69.2\end{array}$

Health status

Number of chronic conditions

$<0.001$

$\begin{array}{lllll}\mathbf{0} & 9,788 & 41.2 & 131,208 & 54.7\end{array}$

$\begin{array}{lllll}1 & 7,632 & 32.1 & 68,868 & 28.7\end{array}$

$\begin{array}{lllll}2 & 3743 & 15.7 & 28,131 & 11.7\end{array}$

$\begin{array}{lllll}\geq 3 & 2616 & 11.0 & 11,496 & 4.8\end{array}$

Heart disease $\quad<0.001$

$\begin{array}{lllll}\text { No } & 18,228 & 76.7 & 214,275 & 89.4\end{array}$

$\begin{array}{lllll}\text { Yes } & 5,551 & 23.3 & 25,428 & 10.6\end{array}$

High blood pressure

$<0.001$

$\begin{array}{lllll}\text { No } & 13,092 & 55.1 & 186,431 & 77.8\end{array}$

$\begin{array}{lllll}\text { Yes } & 10,687 & 45.0 & 53,272 & 22.2\end{array}$

Hyperlipidaemia

$<0.001$

$\begin{array}{lllll}\text { No } & 16,267 & 68.4 & 207,375 & 86.5\end{array}$

$\begin{array}{lllll}\text { Yes } & 7,512 & 31.6 & 32,328 & 13.5\end{array}$

Depression

$<0.001$

$\begin{array}{lllll}\text { No } & 16,720 & 70.3 & 174,994 & 73.0\end{array}$

$\begin{array}{lllll}\text { Yes } & 4,262 & 17.9 & 33,874 & 14.1\end{array}$

Anxiety

$<0.001$

$\begin{array}{lllll}\text { No } & 18,248 & 76.7 & 185,358 & 77.3\end{array}$

Yes

$\begin{array}{llll}2,734 & 11.5 & 23,510 & 9.8\end{array}$

Wellbeing

SF36 (level of limitation)

\section{3,643}

$15.3 \quad 75,231$

31.4

Minor (90-99)

4,615

$19.461,299$

25.6

Moderate (60-89)

6,385

$26.8 \quad 50,387$

21.0

Severe (0-59)

6,866

$28.929,032$

12.1 


\section{Table 1 Demographic, socioeconomic, lifestyle, and health and wellbeing characteristics of participants stratified by diabetes status (Continued)}

\begin{tabular}{|c|c|c|c|c|c|}
\hline \multicolumn{5}{|c|}{ K-10 (level of psychological distress) } & \multirow[t]{2}{*}{$<0.001$} \\
\hline Low (10-15) & 13,934 & 58.6 & 165,517 & 69.1 & \\
\hline Moderate (11-21) & 3,491 & 14.7 & 33,362 & 13.9 & \\
\hline High (22-29) & 1,505 & 6.3 & 11,063 & 4.6 & \\
\hline Very high (30-50) & 797 & 3.4 & 4,307 & 1.8 & \\
\hline \multicolumn{4}{|c|}{ Self-rated general health } & & $<0.001$ \\
\hline Excellent/Very good & 5,910 & 24.9 & 127,369 & 53.1 & \\
\hline Good & 9,638 & 40.5 & 76,297 & 31.8 & \\
\hline Fair/poor & 7,422 & 31.2 & 27,847 & 11.6 & \\
\hline $\begin{array}{l}\text { Self-rated quality } \\
\text { of life }\end{array}$ & & & & & $<0.001$ \\
\hline Excellent/Nery good & 9,082 & 38.2 & 144,058 & 60.1 & \\
\hline Good & 8,482 & 35.7 & 62,099 & 25.9 & \\
\hline Fair/poor & 4,820 & 20.7 & 21,187 & 8.8 & \\
\hline
\end{tabular}

Note: Percentages do not consistently total to $100 \%$ due to missing values.

the aRR for age and obesity was lower among participants with diabetes than those without diabetes (Ratio of RR: 0.74, 95\% CI: $0.70-0.79$ and 0.85 , 95\%CI: $0.80-0.90$ respectively). In contrast, the association between male gender, low income, current tobacco smoking or having anxiety or depression and hospitalisation was stronger in those with diabetes than in those without diabetes. For example, the aRR for gender, current smokers, and selfreport of treatment for anxiety was higher among participants with diabetes than those without diabetes (Ratio of RR: 1.12, 95\% CI: 1.07 - 1.16; 1.14, 95\% CI: $1.04-1.24$; and $1.09,95 \% \mathrm{CI}: 1.03-1.15$ respectively). Interestingly, the associations between the measures of wellbeing such as K10 did not differ significantly between the two groups.

\section{Discussion}

Our study is one of the largest conducted to date to describe the associations between demographic characteristics, socioeconomic status, lifestyle, and health and wellbeing and hospitalisation among community dwelling population with and without diabetes. Compared to those without diabetes, participants with diabetes were more likely to be male, older, born overseas, have lower educational attainment and income, have health risk factors including obesity and physical inactivity, and have poorer health including chronic conditions, hypertension and hyperlipidaemia, and poorer wellbeing. These participant characteristics, together with diabetes, were associated with increased risk of hospitalisation in participants with and without diabetes. Our study also demonstrated that the presence of diabetes could enhance or attenuate these associations.
Study participants with diabetes were $24 \%$ more likely to have a hospital admission for any reason within the year following their recruitment than participants without diabetes and also had more admissions and longer lengths of stay. These findings are variable in relation to a number of previous studies that used different population groups, measures, and follow up periods $[4,27,28]$. Our study used self-reported information to determine diabetes status while other studies used clinical records including admission data $[5,27,28]$, general practice records and/or registers $[4,9,10,27]$, diabetes clinic data [7], and population registers [5] to identify subjects with diabetes. This to our knowledge is the first time that these associations have been reported in a community dwelling healthy population.

Other studies have reported variable proportions of subjects being admitted. Studies among general practice populations in the UK General Practice Research Database observed a lower rate of admission for all patients with diabetes (33.8 per 100 patient years) [4]. The proportion of patients registered with UK general practices admitted in the year of the study was 25\% of those with and 12\% without diabetes [9]; and in an Italian linkage study based on citizens registered with health authorities, the proportion admitted was 19.5\% and $6.4 \% \%$ of patients age $40-65(26.8 \%$ and $15.4 \%$ for those aged more than 64 year) with and without diabetes respectively [10]. As would be expected, larger proportions of subjects were admitted in studies using longer follow up periods: a New Zealand study using general practice data reported that the proportion admitted across a three year study period was $43.5 \%$ and $35.5 \%$ among patients with and without diabetes [27]; an Italian study of patients attending a diabetes clinic reported that $55 \%$ had at least one admission during 4.5 years of follow-up [7]; and a Finnish study where $50.7 \%$ of diabetic patients had at least one hospitalisation during three years of follow-up [5].

Among the participants with diabetes, a diagnostic code indicating a diabetes-related ACSC was recorded for $22 \%$ of participants who were admitted. While this finding was generally consistent with other research, some studies report that diabetes related ACSC admissions contribute to a higher percentage of admissions $[4,5,27,28]$. One explanation for this may be that our community based study population is healthier than the various clinical populations that were employed in comparative studies, that their diabetes is better controlled or they are at an earlier stage of the disease [14]. Other explanations may relate to the definition of diabetes related admissions used [24].

A large number of factors were associated with a self-reported diagnosis of diabetes and with increased risk of hospitalisation in these data. As previously reported, 
Table 2 Summary of hospital admission and length of stay stratified by diabetes status

\begin{tabular}{|c|c|c|}
\hline & Diabetes & No diabetes \\
\hline Number of participants & $\mathrm{N}=23,779$ & $N=239,703$ \\
\hline \multicolumn{3}{|l|}{ Number of admissions } \\
\hline Total admissions & 16,692 & 107,343 \\
\hline day only & 10,231 & 75,197 \\
\hline$>1$ day & 6,460 & 32,145 \\
\hline \multicolumn{3}{|c|}{ Number (\%) of participants admitted } \\
\hline All-cause & $7,807(32.8)$ & $57,970(24.2)$ \\
\hline MCVD $^{1}$ events & $1,034(4.3)$ & $7,752(3.2)$ \\
\hline Diabetes as principal cause & $710(3.0)$ & - \\
\hline Diabetes $\mathrm{ACSC}^{2}$ & $1,744(7.3)$ & - \\
\hline \multicolumn{3}{|c|}{ Rate of hospital admission (age standardised rate per 1,000 participant years) } \\
\hline All-cause & $631.3(624.9-637.7)$ & $454.8(453.0-456.6)$ \\
\hline MCVD events & $86.8(83.2-90.3)$ & $65.8(64.9-66.8)$ \\
\hline Diabetes as principal cause & $49.8(47.0-52.5)$ & - \\
\hline Diabetes ACSC & $136.5(132.2-140.9)$ & - \\
\hline \multicolumn{3}{|c|}{ Rate of hospital admission per participant } \\
\hline \multicolumn{3}{|c|}{ Number of hospital days for all-cause admissions, including day only admissions } \\
\hline \multicolumn{3}{|l|}{ All-cause } \\
\hline Number of participants & 7,807 & 57,970 \\
\hline Mean (SD) & $8.3(18.6)$ & $5.5(12.4)$ \\
\hline Median (Min - Max) & $2(1-472)$ & $1(1-476)$ \\
\hline Interquartile range & $1-8$ & $1-4$ \\
\hline \multicolumn{3}{|c|}{ Length of stay (days) for all-cause admissions, excluding day only admissions } \\
\hline \multicolumn{3}{|l|}{ All-cause } \\
\hline Number of participants & 2,826 & 15,787 \\
\hline Mean (SD) & $8.2(12.2)$ & $7.1(9.9)$ \\
\hline Median (Min, Max) & $4.8(1.2-220)$ & $4(1.1-241)$ \\
\hline Interquartile range & $3-8.6$ & $2.5-7.7$ \\
\hline
\end{tabular}

${ }^{1}$ MCVD: metabolic or cardio-vascular event in principal diagnostic categories.

${ }^{2}$ ACSC: ambulatory care sensitive condition.

older participants and males in our study were more likely to have a hospital admission recorded independently of diabetes status $[4,7,10,29]$. Few studies have explored the impact of socioeconomic status or rural residence on admission to hospital. The authors hypothesised that disadvantaged participants and those living in remote locations would have higher rates of hospitalisation reflecting their poorer access to health care services. Previous studies using hospital data have demonstrated increased all cause, ACSC, and diabetes admissions by disadvantage [30,31]. The present study reported mixed results. The risk of admission was lower for participants residing in areas with relative socioeconomic disadvantage and for participants with low educational attainment. These associations were not influenced by diabetes status. Interestingly, the association between household income and hospitalisation was in the other direction with low household income associated with increased risk of hospitalisation. This may reflect a more direct influence on access to health care than the less precise measure of regional disadvantage. We observed a lower risk of hospitalisation for participants residing in outer regional and remote areas compared to those in major cities. This result is different to previous studies using hospital data that suggest much higher rates of hospitalisation for people living outside major population areas compared to those living in major cities [30,31].

Although the overall rates of cigarette smoking in this study are lower than in the general population $(17.0 \%$ males and $11.8 \%$ for females aged 55-65 years) [32], participants who were current or previous smokers were more likely to have an admission. Our observation of an association 
Table 3 Proportion of participants with a hospital admission, and adjusted relative rates (rate per participant year) and ratios of relative rates of hospital admission (all cause) by demographic, socioeconomic, lifestyle, and health and wellbeing characteristics among participants with and without diabetes

\begin{tabular}{|c|c|c|c|c|c|c|}
\hline \multirow[t]{2}{*}{ Characteristic } & \multicolumn{2}{|l|}{ Diabetes } & \multicolumn{2}{|l|}{ No diabetes } & \multicolumn{2}{|l|}{ Comparison of aRRs } \\
\hline & $\%$ admitted & $\mathrm{aRR}^{*}(95 \% \mathrm{CI})$ & $\%$ admitted & $\operatorname{aRR}^{*}(95 \% \mathrm{Cl})$ & Ratio of RR $(95 \% \mathrm{Cl})$ & p-value \\
\hline \multicolumn{7}{|c|}{ Demographic characteristics } \\
\hline \multicolumn{7}{|l|}{ Gender } \\
\hline Female & 31.5 & 1 & 22.9 & 1 & 1 & \\
\hline Male & 33.9 & $1.16(1.12-1.21)$ & 25.8 & $1.04(1-02-1-05)$ & $1.12(1.07-1.16)$ & $<0.001$ \\
\hline \multicolumn{7}{|c|}{ Age group (years) } \\
\hline $45-59$ & 23.8 & 1 & 17.2 & 1 & 1 & \\
\hline $60-74$ & 32.5 & $1.45(1.37-1.53)$ & 26.9 & $1.65(1.62-1.68)$ & $0.88(0.83-0.93)$ & $<0.001$ \\
\hline$\geq 75$ & 43.7 & $1.72(1.63-1.82)$ & 39.2 & $2.31(2.27-2.36)$ & $0.74(0.70-0.79)$ & $<0.001$ \\
\hline \multicolumn{7}{|l|}{ Country of birth } \\
\hline Australia & 33.8 & 1 & 24.6 & 1 & 1 & \\
\hline Overseas & 30.3 & $0.91(0.87-0.95)$ & 22.9 & $0.94(0.92-0.95)$ & $0.97(0.92-1.01)$ & 0.2 \\
\hline
\end{tabular}

Socioeconomic status

\section{Education}

University

Trade/Cert/Diploma

At least year 10

Less than Year 10

Household income (\$AUD)
$\$ 70,000+$

$\$ 40,000-\$ 69,999$

$\$ 20,000-\$ 39,999$

$<\$ 20,000$

ARIA+

Major Cities

Inner Regional

Outer regional/Remote

SEIFA IRSD (quintiles)

(least disadvantaged) $1 \quad 35.9$

$2 \quad 31.6$

$3 \quad 32.4$

$\begin{array}{ll}4 & 32.7\end{array}$

(most disadvantaged) $5 \quad 32.2$

Lifestyle risk factors

\section{Current tobacco smoking status}

$\begin{array}{ll}\text { Never smoked } & 30.9 \\ \text { Ex-smoker } & 35.6 \\ \text { Current } & 29.6\end{array}$

Alcohol (standard drinks/week)

0

1-6

7-13

\section{1}

0.97(0.91-1.03)

0.89(0.84-0.95)

0.93(0.87-0.99)

1

1.07(0.98-1.17)

1.10(1.01-1.19)

$1.17(1.08-1.26)$

1

0.90(0.87-0.94)

0.86(0.82-0.91)

1

0.76(0.71-0.81)

0.85(0.80-0.90)

$0.78(0.74-0.83)$

0.83(0.77-0.88)

\section{1}

1.14(1.10-1.19)

1.16(1.07-1.25)

\section{1}

0.91(0.87-0.96)

0.85(0.80-0.91)
23.2

26.5

20.5

25.6

22.9

20.9

24.0

24.9

29.0

19.5

21.4

25.4

29.0

25.3

23.6

22.7

26.4

23.1

24.1

23.3

23.9

23.4

\section{1}

1.00(0.98-1.02)

0.98(0.96-1.00)

1.02(0.99-1.04)

1

1.02(1.00-1.05)

1.02(0.99-1.05)

$1.04(1.02-1.07)$

1

0.85(0.84-0.87)

$0.77(0.76-0.79)$

1

0.87(0.85-0.89)

0.81(0.80-0.83)

0.77(0.75-0.79)

0.80(0.78-0.82)

1

1.13(1.11-1.14)

1.02(0.98-1.05)

1.01(0.96-1.05)

0.4

$1.14(1.04-1.24)$

1

0.95(0.90-1.00)

0.94(0.88-1.01)

$0.96(0.94-0.97)$

0.90(0.88-0.92)
0.4

$<0.001$

0.01

0.3

0.09

$<0.001$

0.01

$<0.001$

$<0.001$

0.1

0.7

0.3 
Table 3 Proportion of participants with a hospital admission, and adjusted relative rates (rate per participant year) and ratios of relative rates of hospital admission (all cause) by demographic, socioeconomic, lifestyle, and health and wellbeing characteristics among participants with and without diabetes (Continued)

\begin{tabular}{|c|c|c|c|c|c|c|}
\hline $14-20$ & 33.6 & $0.93(0.86-1.00)$ & 24.6 & $0.94(0.92-0.96)$ & $0.99(0.91-1.07)$ & 0.8 \\
\hline$\geq 20$ & 30.5 & $0.89(0.82-0.97)$ & 23.8 & $0.92(0.89-0.95)$ & $0.97(0.88-1.06)$ & 0.5 \\
\hline \multicolumn{7}{|l|}{ BMI $\left(\mathbf{k g} / \mathbf{m}^{2}\right)$} \\
\hline Normal weight $(<25)$ & 34.1 & 1 & 22.9 & 1 & 1 & \\
\hline Over weight (25-29) & 32.5 & $0.91(0.86-0.95)$ & 24.3 & $1.05(1.03-1.07)$ & $0.87(0.82-0.91)$ & $<0.001$ \\
\hline Obese $(\geq 30)$ & 32.2 & $1.06(1.01-1.12)$ & 25.8 & $1.25(1.22-1.27)$ & $0.85(0.80-0.90)$ & $<0.001$ \\
\hline \multicolumn{7}{|l|}{ Physical activity** } \\
\hline Sufficient & 30.0 & 1 & 22.6 & 1 & 1 & \\
\hline Insufficient & 33.5 & $1.17(1.11-1.22)$ & 25.8 & $1.20(1.17-1.22)$ & $0.98(0.93-1.03)$ & 0.3 \\
\hline Sedentary & 41.9 & $1.38(1.31-1.46)$ & 31.7 & $1.36(1.33-1.39)$ & $1.01(0.96-1.08)$ & 0.6 \\
\hline
\end{tabular}

Health status

Number of chronic conditions

None 24.9

$1 \quad 35.6$

$2 \quad 40.0$

$\geq 3 \quad 44.4$

Heart disease

No

Yes

High blood pressure

No

Hyperlipidaemia

No

Yes

Depression

No

Yes

Anxiety

No

Yes

Wellbeing

SF36 (level of limitation)

No (100)

Minor (90-99)

Moderate (60-89)

Severe (0.59)

K-10 (level of distress)

Low (10-15)

Moderate (16-21)

High (22-29)

Very high (30-50)

24.9

1

1.32(1.26-1.38)

1.56(1.48-1.65)

1.94(1.84-2.06)

$1.34(1.29-1.40)$

22.6

37.2

\section{1}

1.05(1.01-1.09)

22.5

29.9

1

1.03(0.99-1.07)

23.4

29.1

$1 \quad 23.8$

1.43(1.37-1.50)

26.9

1

1.45(1.38-1.53)

24.0

27.1

$1 \quad 16.8$

1.12(1.03-1.22)

21.0

1.67(1.55-1.80)

28.9

$2.31(2.15-2.49)$

39.9

$1.21(1.15-1.28)$

22.5

$1.49(1.38-1.60)$

24.6

26.8

1.99(1.83-2.16)
1.30(1.27-1.32)

1.55(1.52-1.58)

1.85(1.80-1.90)

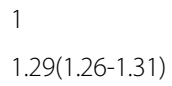

1.04(0.99-1.09)

0.10

0.92(0.88-0.96)

$<0.001$

1.14(1.12-1.16)

1

0.94(0.90-0.98)

$<0.001$

1.10(1.08-1.12)$$
1
$$

1.05(1.00-1.10)

0.04

1.36(1.33-1.38)$$
1
$$

1.33(1.30-1.36)

1.09(1.03-1.15)

$<0.001$
1.25(1.22-1.28)

1.76(1.72-1.80)

2.43(2.37-2.49)

$0.90(0.82-0.98)$

0.95(0.88-1.03)

0.95(0.88-1.03)

0.2

0.2

1

1.22(1.19-1.25)

0.99(0.94-1.05)

0.8

1.39(1.35-1.44)

1.07(0.99-1.16)

0.09

1.83(1.75-1.91)

1.09(0.99-1.19) 
Table 3 Proportion of participants with a hospital admission, and adjusted relative rates (rate per participant year) and ratios of relative rates of hospital admission (all cause) by demographic, socioeconomic, lifestyle, and health and wellbeing characteristics among participants with and without diabetes (Continued)

\begin{tabular}{|c|c|c|c|c|c|c|}
\hline \multicolumn{7}{|c|}{ Self-rated general health } \\
\hline Excellent/Very good & 24.9 & 1 & 19.6 & 1 & 1 & \\
\hline Good & 31.2 & $1.35(1.27-1.43)$ & 26.7 & $1.40(1.37-1.42)$ & $0.96(0.91-1.02)$ & 0.2 \\
\hline Fair/poor & 40.6 & $1.98(1.87-2.10)$ & 36.8 & 1.95(1.91-1.99) & $1.02(0.96-1.08)$ & 0.6 \\
\hline \multicolumn{7}{|c|}{ Self-rated quality of life } \\
\hline Excellent/Very good & 27.3 & 1 & 20.9 & 1 & 1 & \\
\hline Good & 33.1 & $1.28(1.22-1.34)$ & 27.5 & $1.27(1.25-1.29)$ & $1.01(0.96-1.06)$ & 0.8 \\
\hline Fair/poor & 41.3 & $1.59(1.52-1.68)$ & 34.8 & $1.69(1.65-1.73)$ & $0.94(0.89-0.99)$ & 0.03 \\
\hline
\end{tabular}

${ }^{*}$ Adjusted for age and gender;

**Physical activity: Sufficient: $>=150$ minutes of walking and > =5 sessions per week; Sedentary: no physical activity reported; and Insufficient: all other categories.

between obesity and physical inactivity and risk of hospitalisation has been observed elsewhere [7,33]. The observed relatively poor health status of study participants with diabetes and the observed association of health status with increased risk of hospitalisation for all participants has been previously reported $[4,7,27,29]$. The interesting finding was the attenuation of the association with increased hospitalisation for obesity, high blood pressure and hyperlipidaemia among participants with diabetes and enhancement of hospitalisation admission among those who had been treated for anxiety and depression. These effects were not observed for the wellbeing measures including K-10.

The differences in the observed strength of the associations between the risk factors examined and risk of hospitalisation for participants with and without diabetes may be explained by the presence of diabetes, the potential for which is suggested in our conceptual framework (Figure 1). These differences were supported by statistically significant interaction terms and changes demonstrated by the ratio of $R R$. The ratio of RR is a robust means of comparing effect sizes in subgroup analysis and may be more informative than the use of interaction terms. [26] Firstly, male participants were over-represented among participants with diabetes and male participants with diabetes were significantly more likely to be hospitalised than those without diabetes. Not only were a greater proportion of younger participants with diabetes admitted than those without diabetes, the association between age and hospital admission was attenuated for participants with diabetes. This suggests that diabetes may have an "ageing effect" that leads to poorer health at an earlier age for those with diabetes compared to those without diabetes. Once adjusted for age and gender, the patterns of socioeconomic factors influencing the relative rate of hospitalisation were broadly similar (same direction and pattern of factors) for participants with and without diabetes (Table 3). However, participants from rural and remote locations were more likely to have a hospital admission when diabetes was present than when it was not, even though the stratified relative rates indicated that both groups had a lower risk of hospitalisation. While hospitalisation was more common among participants with low income in general, the association between low income and hospitalisation was enhanced among participants with diabetes.

In the stratified analysis, the effects of obesity, hypertension and hyperlipidaemia on the likelihood of hospital admission were reduced in participants with diabetes. One possible interpretation of this result may be that the impact of diabetes on the risk of admission especially for cardiovascular health may be mediated in part by the physiological changes associated with obesity, hypertension and hyperlipidaemia. Thus, when diabetes is accounted for in the modelling, there is little increase in risk of hospitalisation that can be accounted for by these other factors. Further investigation of these relationships is warranted. This finding differs from that of a previous study which showed an enhanced association between hypertension and hospitalisation in people with diabetes [34]. In contrast, the association between male gender, low income, smoking, anxiety or depression and the risk of hospitalisation was enhanced in people with diabetes. This may be due to a variety of factors including synergistic effects of risk factors such as smoking and diabetes on the risk of cardiovascular disease. There may also be synergy in the accessibility of services. For example, the financial burden on patients with diabetes in accessing health services and employment, may exacerbate the effect of low incomes on health and the pattern of health service use. The enhancement of the association between anxiety and depression and hospitalisation has been observed previously [35]. In our study we found that self-report of treatment for these conditions was enhanced among participants with diabetes while there was no modification for $\mathrm{K}-10$ scores, a screening tool for measuring current distress. Poorer health outcomes have also been observed for adults with both diabetes and anxiety and/or depression including higher rates of myocardial infarction [36], and symptom control [37,38]. 


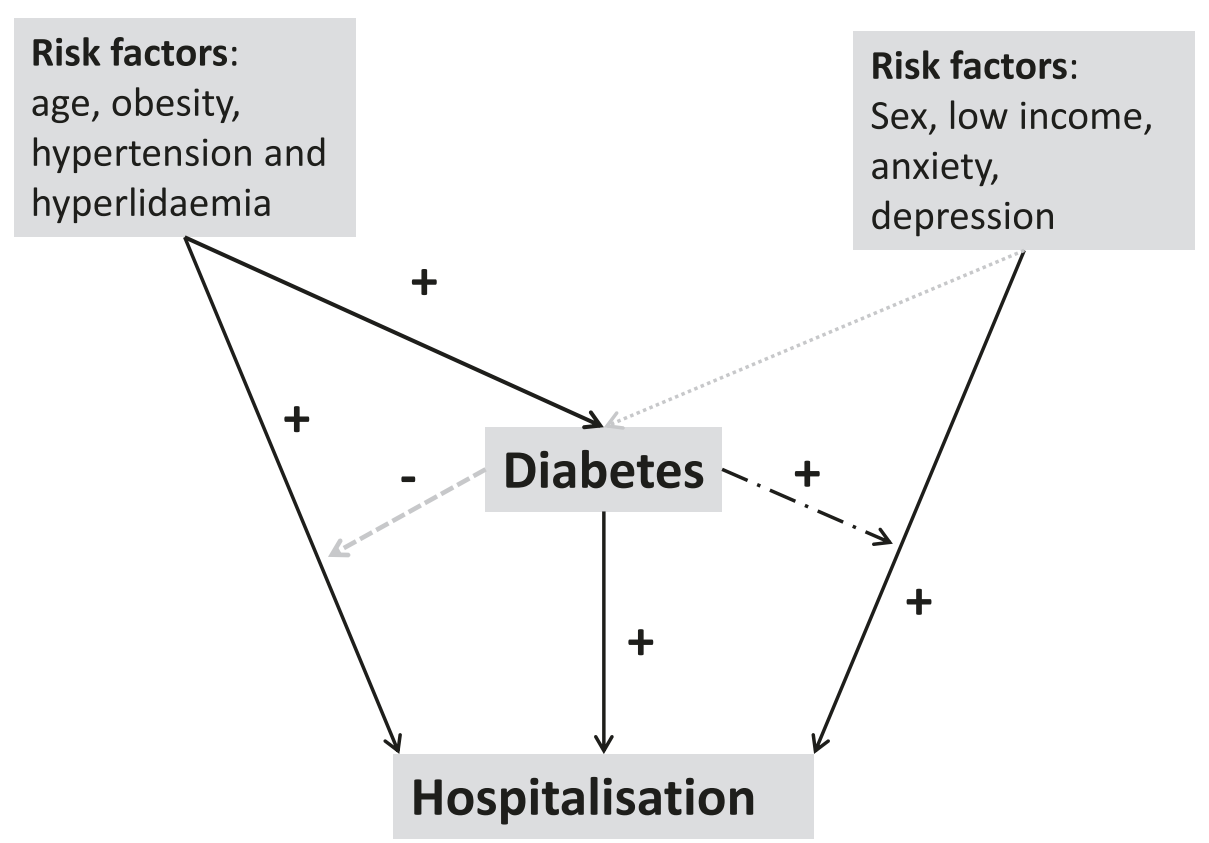

Figure 1 conceptual framework to explain the impact of diabetes on the associations between other explanatory factors and hospitalisation. Coding: solid black line: positive association; dashed/dot black line: diabetes enhances the association between risk factors and hospitalisation; dotted grey line: a weak positive association; dashed grey line: diabetes associated with attenuation of association.

\section{Implications for health service provision especially primary care}

This study confirms the association between the presence of diabetes and increased rates of hospitalisation. It provides new information on the impact of important risk factors that are associated with both the prevalence of diabetes and higher rates of hospitalisation among people with diabetes. Importantly diabetes mediates some associations between risk factors and hospitalisation with enhancement of some associations and attenuation of others. Although the observed associations between obesity, hypertension and hyperlipidaemia and hospitalisation were reduced after stratification for diabetes, the higher rates of these factors indicates that their control remains important in the prevention of diabetes and of complications and hospitalisation in people with diabetes. The apparent enhancement of the associations between male gender, low income, smoking, and anxiety and depression is and hospitalisation among participants with diabetes is of considerable concern. Males and smokers are lower users of primary care suggesting that a more proactive approach to encourage their use of primary care may be required if rates of admission to hospital are to be lowered. Conversely low income participants and those with anxiety and depression are more frequent users of primary care suggesting that more support for self-management and low cost (to consumer) alternatives to traditional medical care may be more appropriate for these groups.
This has direct implications for community education, practice support and quality improvement programs in the development of primary care organisations such as Medicare Locals in Australia, as well as initiatives by diabetes organisations.

\section{Strengths and limitations}

This is one of the largest population based studies of the impact of diabetes on hospitalisation undertaken. Few studies have had the opportunity to link a large population data collection to temporal data in terms of the use of services such as hospitals. A further strength of this study was the availability of comprehensive information about the demographic characteristics, socioeconomic status, lifestyle, and health and wellbeing of participants. These data and identification of the sub-group with diabetes depended on information provided at the baseline survey and we have previously demonstrated that the use of self-report data is an acceptable means of identification [15]. However the linked data used in this study did not include biological information that would have provided confirmatory diagnostic evidence of diabetes status or control of biological indicators of diabetes such as $\mathrm{HbA1c}$ levels that have been reported in other studies $[7,10,39]$. The study could not differentiate between type 1 and type 2 diabetes and was thus not able to explore whether type of diabetes is an important predictor of hospitalisation.. The studies reported were all carried 
out under different health systems which may have been influenced by different payment systems, admission and discharge policies, and timeliness of data [40].

\section{Conclusions}

This study is one of the few studies published to explore the impact of diabetes on hospitalisation in a large nonclinical population, the 45 and Up Study. Using record linkage, the study demonstrated that participants were $24 \%$ more likely to have a hospital admission in the year following recruitment if they reported a diagnosis of diabetes than if they did not. Admitted participants with diabetes had more admissions and longer length of stay than participants without diabetes. Further this study was one of the few to explore the association between a comprehensive set of demographic factors, socioeconomic status, lifestyle and health factors on hospitalisation and associated impact of the presence of a diagnosis of diabetes. Although age and obesity are both major risk factors for diabetes, this study is one of the first to demonstrate attenuation of the associations between both age and obesity and risk of hospitalisation by the presence of diabetes. The increased associations between hospitalisation and other risk factors for hospitalisation such as gender and low income in participants with diabetes may be explained by their synergistic influence on health status and the way services are accessed.

\section{Competing interests}

The authors declare that they have no competing interests.

\begin{abstract}
Authors' contributions
EC: is the study leader. She conceptualised the study, led the design and implementation of the study, oversaw data analysis and wrote this manuscript. $\mathrm{MH}$ : is a senior member of the research group. He has contributed extensively to the study design, implementation, and interpretation of the analysis and writing of the manuscript. MDF: Islam was the study project officer. He participated in the implementation of the study, performed the statistical analysis and helped to draft the manuscript. DTT: was a member of the research team. She participated in the implementation of the study, contributed to the statistical analysis and helped to draft the manuscript. BJ: is a senior member of the research team and has contributed to the development and implementation of the study and the preparation of this manuscript. LJ: is a senior member of this research group and is a member of the 45 and Up Study Research Team. She has contributed to the development and implementation of the research and contributed to the preparation of this manuscript. JF: is a senior member of the research group. He has provided advice on the development of the research, classification of the study variables, and contributed to the implementation of the study. $\mathrm{MH}$ : was a senior member of the research group. She provided expert advice on economic aspects of the research. She was a member of the study advisory group and has made a significant contribution to the preparation of this paper. All authors read and approved the final manuscript.
\end{abstract}

\section{Acknowledgements}

The authors wish to thank the NSW residents participating in the 45 and Up Study for enabling this research. The 45 and Up Study is managed by the Sax Institute in collaboration with Study Partners: Cancer Council New South Wales; the New South Wales Division of the National Heart Foundation of Australia; the New South Wales Department of Health; beyond blue: the national depression initiative; and the New South Wales Department of
Ageing, Disability and Home Care. The authors would also like to thank the NSW Ministry of Health for access to Admitted Patient Data Collection, the NSW Register of Births Deaths and Marriages for access to death data, and the Centre for Health Record Linkage for facilitating the record linkage. This project was supported by a National Health and Medical Research Council (NHMRC) project grant (APP630498). Funding sources had no role in study design; in the collection, analysis, and interpretation of data; in the writing of the report; nor in the decision to submit the article for publication. The authors would also like to acknowledge Nayyereh Aminisani for assistance with the literature search.

\section{Author details}

${ }^{1}$ Centre for Primary Health Care and Equity, University of New South Wales, Sydney, NSW 2052, Australia. ${ }^{2}$ Centre for Big Data Research in Health, University of New South Wales, Sydney, NSW 2052, Australia. ${ }^{3}$ Centre for Research, Evidence Management and Surveillance, Sydney and South Western Sydney Local Health Districts, Locked Bag 7017, Liverpool, NSW 1871, Australia. ${ }^{4}$ School of Public Health and Community Medicine, University of New South Wales, Sydney 2052, Australia. ${ }^{5}$ Centre for Health Research, School of Medicine, University of Western Sydney, Locked Bag 1797, Penrith, NSW 2751, Australia. ${ }^{6}$ Bankstown-Lidcombe Hospital, Eldridge Road, Bankstown, NSW 2200, Australia. ${ }^{7}$ Centre for Health Economics Research and Evaluation, Faculty of Business, University of Technology, Sydney, PO Box 123, Broadway, NSW 2007, Level 4, 645 Harris Street, Ultimo, NSW 2007, Australia.

Received: 21 July 2014 Accepted: 16 December 2014

Published online: 22 January 2015

\section{References}

1. Australian Institute of Health and Welfare. Diabetes prevalence in Australia. An assessment of national data sources. Canberra: AlHW; 2009.

2. Renders CM, Valk GD, Griffin SJ, Wagner EH, Van Eijk JT, Assendelft WJ. Interventions to improve the management of diabetes in primary care, outpatient, and community settings: a systematic review. Diabetes Care. 2001;24(10):1821-33.

3. Kasper D, Braunwald E. Hauser S. In: Kasper D, New HT, York NY, editors. Harrison's principles of internal medicine. 16th ed. London: McGraw-Hill Medical Publishing Division; 2004

4. Khalid JM, Raluy-Callado M, Curtis BH, Boye KS, Maguire A, Reaney M. Rates and risk of hospitalisation among patients with type 2 diabetes: retrospective cohort study using the UK General Practice Research Database linked to English Hospital Episode Statistics. Int J Clin Pract. 2013;68(1):40-80.

5. Aro S, Kangas T, Reunanen A, Salinto M, Koivisto V. Hospital use among diabetic patients and the general population. Diabetes Care. 1994;17(11):1320-9.

6. Colagiuri S, Dickinson S, Girgis S, Colagiuri R. National Evidence Based Guideline for Blood Glucose Control in Type 2 Diabetes. Canberra: Diabetes Australia and the NHMRC; 2009.

7. Bo S, Ciccone G, Grassi G, Gancia R, Rosato R, Merletti F, et al. Patients with type 2 diabetes had higher rates of hospitalization than the general population. J Clin Epidemiol. 2004;57(11):1196-201.

8. Rosenthal MJ, Fajardo M, Gilmore S, Morley JE, Naliboff BD. Hospitalization and mortality of diabetes in older adults. A 3-year prospective study. Diabetes Care. 1998;21(2):231-5.

9. Donnan PT, Leese GP, Morris AD, Diabetes A. Research in Tayside, Scotland Medicine Monitoring Unit Collaboration: Hospitalizations for people with type 1 and type 2 diabetes compared with the nondiabetic population of Tayside, Scotland: a retrospective cohort study of resource use. Diabetes Care. 2000;23(12):1774-9.

10. De Berardis G, D’Ettorre A, Graziano G, Lucisano G, Pellegrini F, Cammarota $\mathrm{S}$, et al. The burden of hospitalization related to diabetes mellitus: a population-based study. Nutr Metab \& Cardiovasc Dis. 2012;22(7):605-12.

11. Carral F, Olveira G, Salas J, Garcia L, Sillero A, Aguilar M. Care resource utilization and direct costs incurred by people with diabetes in a Spanish hospital. Diabetes Res \& Clin Prac. 2002;56(1):27-34.

12. Panser LA, Naessens JM, Nobrega FT, Palumbo PJ, Ballard DJ. Utilization trends and risk factors for hospitalization in diabetes mellitus. Mayo Clin Proc. 1990;65(9):1171-84.

13. Burke $V$, Zhao $Y$, Lee AH, Hunter E, Spargo RM, Gracey M, et al. Predictors of type 2 diabetes and diabetes-related hospitalisation in an Australian Aboriginal cohort. Diabetes Res \& Clin Pract. 2007;78(3):360-8. 
14. 45 and Up Study Collaborators, Banks E, Redman S, Jorm L, Armstrong B, Bauman A, et al. Cohort profile: the 45 and up study. Int J Epidemiol. 2008;37(5):941-7.

15. Comino E, Tran DT, Haas M, Flack J, Jalaludin B, Jorm L, et al. Validating self-report of diabetes and diabetes medication use by participants in the 45 and Up study: a record linkage study. BMC Health Serv Res. 2013;13(1):481.

16. Australian Institute of Health and Welfare A. The Active Australia Survey: a guide and manual for implementation, analysis and reporting. Canberra: AlHW; 2003

17. Australian Bureau of Statistics: Information Paper: An Introduction to Socio-Economic Indexes for Areas (SEIFA). Paper no. 2039.0 Canberra: ABS; 2008. Available at: http://www.abs.gov.au/websitedbs/censushome.nsf/ home/seifa Accessed 3 November 2014

18. Australian Institute of Health and Welfare. 2004. Rural, regional and remote health: a guide to remoteness classifications. AlHW cat. no. PHE 53. Canberra: AlHW. Available at [http://www.aihw.gov.au/publication-detail/? id=6442467589] Accessed 3 November 2014

19. Kessler R Mroczek D: Final Version of our Non-Specific Psychological Distress Scale [memo dated 3/10/94]. Ann Arbor (MI) Survey Research Center of the Institute for Social Research, University of Michigan; Michigan 1994

20. McCallum J. The SF-36 in an Australian Sample: validation of a new, generic health status measure. Aust J Public Health. 1995;19(2):160-6.

21. Jorm LR, Shepherd LC, Rogers KD, Blyth FM, Jorm LR, Shepherd LC, et al. Smoking and use of primary care services: findings from a population-based cohort study linked with administrative claims data. BMC Health Serv Res. 2012;12:263

22. Centre for Health Record Linkage. The first three years 2006-07 to 2008-09. Sydney: Centre for Health Record Linkage (CHeReL); 2009.

23. Kelman CW, Bass AJ, Holman CD. Research use of linked health data-a best practice protocol. Aust N Z J Public Health. 2002;26(3):251-5.

24. Australian Institute of Health and Welfare: National Healthcare Agreement: PI 22-Selected potentially preventable hospitalisations, 2011 Canberra: AlHW. Available at: http://meteor.aihw.gov.au/content/index.phtml/itemld/448108 Accessed 3 November 2014

25. Lee AH, Wang K, Scott JA, Yau KK, McLachlan GJ. Multi-level zero-inflated poisson regression modelling of correlated count data with excess zeros. Stat Methods Med Res. 2006;15(1):47-61.

26. Altman D, Bland JM. Interaction revisited: the difference between two estimates. Br Med J. 2003;326(7382):219.

27. Tomlin AM, Tilyard MW, Dovey SM, Dawson AG. Hospital admissions in diabetic and non-diabetic patients: a case-control study. Diabetes Res \& Clinical Pract. 2006;73(3):260-7.

28. Kim S, Boye KS. Excessive hospitalizations and its associated economic burden among people with diabetes in the United States. Value Health. 2009;12(2):267-72.

29. Moss SE, Klein R, Bek K. Risk factors for hospitalisation in people with diabetes. Arch Intern Med. 2012;159:2053-7.

30. Ansari Z, Haider SI, Ansari H, de Gooyer T, Sindall C. Patient characteristics associated with hospitalisations for ambulatory care sensitive conditions in Victoria, Australia. BMC Health Serv Res. 2012;12:475.

31. Australian Institute of Health and Welfare, O'Brien K, Thow AM, Ofei S. Diabetes hospitalisations in Australia 2003-2004. In: Bulletin no. 47, Cat no. 84. Canberra: AlHW; 2006.

32. Centre for Epidemiology and Research. The health of the people of New South Wales - Report of the Chief Health Officer. Summary Report, 2010. Sydney: NSW Department of Health; 2010.

33. Korda RJ, Liu B, Clements MS, Bauman AE, Jorm LR, Bambrick HJ, et al. Prospective cohort study of body mass index and the risk of hospitalisation: findings from 246361 participants in the 45 and Up Study. Int J Obes (Lond). 2013;37:790-9.

34. Lin M, Chen Y, Sigal RJ. Impacts of diabetes and hypertension on the risk of hospitalization among less educated people. J Hum Hypertens. 2007;21(3):225-30

35. Stewart SM, Rao U, Emslie GJ, Klein D, White PC. Depressive symptoms predict hospitalization for adolescents with type 1 diabetes mellitus. Pediatrics. 2005;115(5):1315-9.

36. Scherrer JF, Garfield LD, Chrusciel T, Hauptman PJ, Carney RM, Freedland KE, et al. Increased risk of myocardial infarction in depressed patients with type 2 diabetes. Diabetes Care. 2011;34(8):1729-34.
37. Anderson RJ, Grigsby AB, Freedland KE, de Groot M, McGill JB, Clouse RE, et al. Anxiety and poor glycemic control: a meta-analytic review of the literature. Int J Psychiatry Med. 2002;32(3):235-47.

38. Lawrence JM, Standiford DA, Loots B, Klingensmith GJ, Williams DE, Ruggiero A, et al. Prevalence and correlates of depressed mood among youth with diabetes: the SEARCH for Diabetes in Youth study. Pediatrics. 2006;117(4):1348-58.

39. Moss SE, Klein R, Klein BE. Risk factors for hospitalization in people with diabetes. Arch Intern Med. 1999;159(17):2053-7.

40. Metcalfe C, Thompson SG, Cowie MR, Sharples LD: The use of hospital admission data as a measure of outcome in clinical studies of heart failure. European Heart Journal, 24(1):105-112

\section{Submit your next manuscript to BioMed Central and take full advantage of:}

- Convenient online submission

- Thorough peer review

- No space constraints or color figure charges

- Immediate publication on acceptance

- Inclusion in PubMed, CAS, Scopus and Google Scholar

- Research which is freely available for redistribution 